\title{
Age-Related Alterations in Endothelial Function of Femoral Artery in Young SHR and WKY Rats
}

\author{
Angelika Puzserova, ${ }^{1}$ Veronika Ilovska, ${ }^{1,2}$ Peter Balis, ${ }^{1}$ Peter Slezak, ${ }^{1}$ and Iveta Bernatova ${ }^{1}$ \\ ${ }^{1}$ Institute of Normal and Pathological Physiology, Centre of Excellence for Examination of Regulatory Role of Nitric Oxide in \\ Civilization Diseases, Slovak Academy of Sciences, Sienkiewiczova 1, 81371 Bratislava, Slovakia \\ ${ }^{2}$ Institute of Medical Biochemistry, Jessenius Faculty of Medicine, Comenius University, Mala Hora 4, 03601 Martin, Slovakia
}

Correspondence should be addressed to Angelika Puzserova; angelika.puzserova@savba.sk

Received 12 December 2013; Accepted 10 February 2014; Published 20 March 2014

Academic Editor: Ramaroson Andriantsitohaina

\begin{abstract}
Copyright (C) 2014 Angelika Puzserova et al. This is an open access article distributed under the Creative Commons Attribution License, which permits unrestricted use, distribution, and reproduction in any medium, provided the original work is properly cited.

The present study was designed to evaluate the effects of vascular aging in juvenescence on endothelial function in femoral arteries and to assess differences between normotensive and hypertensive rats. The aim of the study was to determine if age affected nitric oxide- (NO-) mediated relaxations in normotensive and hypertensive rats. Juvenile (7-week-old) and young adult (22-week-old) male Wistar-Kyoto (WKY) and spontaneously hypertensive rats (SHR) were used in this study. Femoral artery (FA) reactivity was determined by wire myograph and NO synthase activity by conversion of $\left[{ }^{3} \mathrm{H}\right]$-L-arginine. During juvenescence systolic blood pressure (tail-cuff) increased significantly only in SHR, while NO synthesis decreased significantly in both strains. Endotheliumdependent relaxations to acetylcholine were reduced in the FA of SHR compared to age-matched WKY at both ages, yet these parameters were unchanged in adult rats compared with juvenile animals. The NO-dependent component of vasorelaxation was markedly reduced, whereas the NO-independent component was increased in adult compared to juvenile rats in both strains. The endothelial dysfunction in SHR at both ages was associated with reduction of NO-independent mechanisms. In conclusion, aging in early periods of life was associated with reduction of vascular NO production and bioavailability in both strains investigated. This reduction was however fully compensated by accentuation of NO-independent mechanisms.
\end{abstract}

\section{Introduction}

Studies using experimental animal models as well as clinical research have indicated that hypertension is associated with endothelial dysfunction, a state in which the endothelial disorder leads to reduced vasodilation and increased vasoconstriction. Despite enormous research effort, the causal relationship between endothelial dysfunction and hypertension has remained unclear.

Endothelial dysfunction develops due to various risk factors, including aging of the organism. The latter, however, may be the causative agent even in the absence of established risk factors $[1,2]$. Moreover, aging and hypertension have been identified as major risk factors for cardiovascular diseases. Advanced aging is associated with reduced endothelium-dependent relaxations, that is, endothelial dysfunction, in both human and animal arteries, promoting thus the initiation and development of cardiovascular diseases [38].

Vasorelaxation is primarily controlled by the endothelium, and both by production of endothelium-derived relaxing factors (EDRFs), as nitric oxide (NO), endotheliumderived hyperpolarizing factor (EDHF) and prostacyclin $\left(\mathrm{PGI}_{2}\right)$, and also by endothelium-derived contracting factors (EDCFs), which reduce vasorelaxation [9]. Disbalance between EDRFs and EDCFs results in an endothelial dysfunction, which has been observed in various diseased states, including hypertension [10]. Increased EDCFs-induced vasoconstriction, mediated presumably by cyclooxygenase (COX) metabolites, participates in impairment of endotheliumdependent vasorelaxation in both genetic models of arterial hypertension and age-induced vascular changes $[5,6,10]$.

Regarding vasorelaxation, $\mathrm{NO}$ mediates many physiological and pathophysiological functions. Age-related vascular 
dysfunction was shown to result mainly from reduced NO production $[11,12]$. In human umbilical vein endothelial cells, aging decreased the production of $\mathrm{NO}$ and the activity and expression of eNOS protein [11]. Several studies indicated that aging blunted NO-dependent relaxations [8, 13-16]. All the reported studies, however, investigated vascular function of middle-aged and old (55-week- to 35-month-old) rats compared to young adult (12- to 24 -week-old) rats. According to animal models and the vascular bed studied, agingrelated changes can involve different mechanisms. Yet little information is available on early alterations in the NOmediated vasorelaxation from blood vessels of juvenile and young adult rats either with or without a genetic load of hypertension.

As alterations in aging may, at least in part, depend on hemodynamic factors such as arterial pressure [14], a further aim of this study was to determine the influence of aging in young spontaneously hypertensive rats (SHR). We used SHR as this experimental model of genetic hypertension is similar to the human form of increased blood pressure (BP) and can help to better understand the mechanisms of essential hypertension in humans $[17,18]$. The characteristic acceleration of BP rise in SHR (as compared with normotensive WKY rats) occurs mainly between the 3rd and 10th week of age. Over this period of age, their BP rapidly increases and continues to rise at least until the age of 20 weeks [19]. In contrast, the BP of WKY reaches adult levels by the 10th week of age [19]. High blood pressure in SHR may result from sympathetic hyperactivity and sympathetic vasoconstriction [20, 21]. In SHR, the femoral artery, a conductance medium-size artery, exhibits increased vasoconstriction to the sympathetic vasoconstrictor noradrenaline [22-24] and endothelial dysfunction in adulthood [25]. However, opposite findings were also described in the SHR femoral artery [26]. Nevertheless, pharmacological studies using the femoral artery are highly relevant for a better understanding of the pathophysiology of peripheral artery disease (PAD), whose incidence has an increasing tendency in the world population $[27,28]$ similar to hypertension. Many factors, including hypertension and advancing age, have been implicated in the pathogenesis of PAD $[27,29,30]$. Although the femoral arteries are not the main factor contributing to elevated peripheral resistance in hypertension, altered vascular function in early periods of life may be involved in various diseased states in old age. In addition, different mechanisms may be involved in the vascular aging in the aorta, the common femoral artery [31], and small mesenteric arteries [5] in various periods of life.

In order to determine vascular changes in early periods of life, we investigated alterations in $\mathrm{BP}$, NO production, and vascular function of the isolated femoral artery of juvenile and young adult male normotensive Wistar-Kyoto (WKY) and spontaneously hypertensive rats. In addition, we determined NO-dependent and -independent components of endothelium-dependent relaxation to investigate possible compensatory mechanisms participating in the maintenance of normal vascular function in juvenescence.

\section{Materials and Methods}

2.1. Animals and Experimental Design. Male 7-week-old (juvenile) and 22-week-old (young adult) SHR and WKY rats were used $(n=8-10)$. All the rats used in the present study were born in our certified animal facility (Institute of Normal and Pathological Physiology SAS). The rats were housed in groups of five animals per cage, each strain separately, in an air-conditioned room at constant temperature $\left(22-24^{\circ} \mathrm{C}\right)$ and humidity (45-60\%) at a $12: 12$-h light/dark cycle $(06: 00-$ $18: 00 \mathrm{~h}$ lights on) and they were maintained on a standard pellet diet and tap water ad libitum. All procedures used were approved by the State Veterinary and Food Administration of the Slovak Republic.

Systolic blood pressure (SBP) and heart rate (HR) were determined noninvasively in conscious rats by the tail-cuff method at the end of the experiment as described previously [32]. Body weight (BW) was recorded at the same time. Seven- and 22-week-old rats were killed by decapitation after a brief $\mathrm{CO}_{2}$ anesthesia. Wet weights of the left heart ventricle (LVW) were determined for calculation of their relative weights (LVW/body weight) to evaluate the degree of cardiac hypertrophy.

\subsection{Assessment of Vascular Reactivity of the Femoral Artery by} Wire Myograph. Immediately after decapitation, the femoral artery was carefully excised and cleaned of adipose or connective tissue. The arteries were then cut into segments (1.28 \pm $0.04 \mathrm{~mm}$ long) and mounted as ring-shaped preparations in the Mulvany-Halpern style small vessel wire myograph chamber (Dual Wire Myograph System 410A, DMT A/S, Aarhus, Denmark) to determine the vascular reactivity during isometric conditions as described elsewhere [33]. The preparations were bathed in modified physiological salt solution (PSS) oxygenated with a mixture of $95 \% \mathrm{O}_{2}$ and $5 \%$ $\mathrm{CO}_{2}$ and maintained at $37^{\circ} \mathrm{C}[26,32]$. The composition of the PSS was (in mmol/L) $\mathrm{NaCl} 118.99, \mathrm{KCl} 4.69, \mathrm{NaHCO}_{3} 25$, $\mathrm{MgSO}_{4} \cdot 7 \mathrm{H}_{2} \mathrm{O} 1.17, \mathrm{KH}_{2} \mathrm{PO}_{4} 1.18, \mathrm{CaCl}_{2} \cdot 2 \mathrm{H}_{2} \mathrm{O} 2.5, \mathrm{Na}_{2}$ EDTA 0.03 , and glucose 5.5 ( $\mathrm{pH} 7.4)$. The normalization procedure, the calculations for normalized inner diameter, and the experimental protocol for the femoral artery were described previously $[25,32]$.

Briefly, the contractile response to $125 \mathrm{mmol} / \mathrm{L} \mathrm{KCl}$ (PSS was changed to KPSS in which $\mathrm{NaCl}$ was exchanged for an equimolar concentration of $\mathrm{KCl}$ ) for $2 \mathrm{~min}$ was first obtained on each arterial ring followed by washings with PSS. An equilibration period of $20 \mathrm{~min}$ was allowed between each series of experiments. After confirming a sufficient contractile response to KPSS, experiments with noradrenaline $\left(10^{-5} \mathrm{~mol} / \mathrm{L}\right)$ were started to obtain phasic and tonic contractile responses [24]. Since the arteries must be optimally precontracted to assess relaxation responses, a submaximal tone was induced with $10^{-6} \mathrm{~mol} / \mathrm{L}$ serotonin (Ser) $[15,28]$. This precontraction agent was then used for all subsequent relaxation studies. When the contraction of the femoral artery to Ser reached a steady state, increasing concentrations of the vasodilator acetylcholine (ACh, 0.001 to $10 \mu \mathrm{mol} / \mathrm{L}$ ) were added in cumulative manner to perform endothelium-dependent concentration-response curves [34] 
followed by rinsings. To examine whether $\mathrm{NO}$ was involved in ACh-induced vasorelaxation of the femoral artery, $\mathrm{N}^{\mathrm{G}}$ nitro-L-arginine methyl ester (L-NAME), a nonspecific NOS inhibitor, was added to the PSS at $300 \mu \mathrm{mol} / \mathrm{L}$ and was allowed to incubate for $25 \mathrm{~min}$. In the presence of L-NAME, when ACh-induced NO release could be precluded, the concentration-response curve for $\mathrm{ACh}$ was repeated. The drugs were then washed out (PSS, $30 \mathrm{~min}$ ) and the nitric oxide donor sodium nitroprusside (SNP, 0.001 to $10 \mu \mathrm{mol} / \mathrm{L}$ ) was added in a cumulative fashion to assess an endotheliumindependent, however NO-dependent, vascular relaxation. After the following wash-out, the femoral artery rings were stimulated again with high concentration of $\mathrm{K}^{+}(125 \mathrm{mmol} / \mathrm{L})$ in depolarising solution to induce maximal contraction (PSS was changed to KPSS) and then left to achieve a plateau. The maximal tension reached with this depolarizing solution was set as $100 \%$ to express the active tension generated by noradrenaline [35].

NO-mediated relaxation was determined by measuring the portion of ACh-induced relaxation that was abolished by L-NAME [36, 37]. Calculations were performed by determination of the area under the curve (AUC, in arbitrary units, au) of individual dose-response curves. The NOmediated response was then calculated as the difference between the AUC of ACh-induced relaxation in the absence and presence of L-NAME. The extent of vasorelaxation was expressed in relative values as the percentage of the initial contraction induced by Ser as well as in absolute values $(\mathrm{mN} / \mathrm{mm})$ to minimize a possible effect of different Serinduced precontraction tone $[25,38,39]$. Vasoconstrictions were determined as the maximal tension and they were expressed as active wall tension in $\mathrm{mN} / \mathrm{mm}$. All chemicals used were purchased from Sigma-Aldrich (Germany) and Merck Chemicals (Germany), except noradrenaline hydrogenotartras (Zentiva, Czech Republic).

2.3. Nitric Oxide Synthase Activity. Total NO synthase (NOS) activity was measured in tissue homogenates of the aorta $(200 \mathrm{mg} / \mathrm{mL})$ by determination of $\left[{ }^{3} \mathrm{H}\right]$-L-citrulline formation from $\left[{ }^{3} \mathrm{H}\right]$-L-arginine (MP Biomedicals, USA, 50 $\mathrm{Ci} / \mathrm{mmol}$ ), as described previously [32] and expressed as $\mathrm{pmol} / \mathrm{min} / \mathrm{mg}$ of tissue proteins as determined by the Lowry method [40]. All chemicals used were purchased from SigmaAldrich (Germany) and Merck Chemicals (Germany).

2.4. Statistical Analysis. Data are presented as group mean values \pm SEM of the number $(n)$ of independent measurements. Results were analyzed by analysis of variance (ANOVA). Two-way ANOVA (with age and strain as independent variables) was used to compare basic biometric and cardiovascular parameters and normalized inner diameter, vascular constrictions, and nitric oxide synthase activity. In case of significant results pairwaise comparison with Bonferroni adjustment was employed. Homogeneity of variances and normality of distribution were tested by Levene's test and by Shapiro-Wilk's test, respectively. Concentration response curves were compared using two-way repeated measurements ANOVA, followed by vertical contrast with

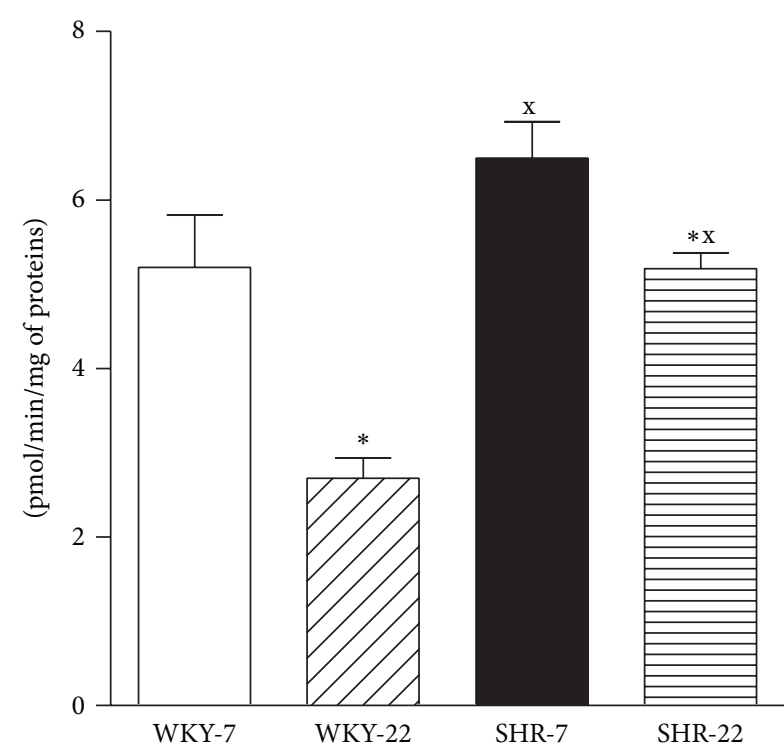

FIGURE 1: Nitric oxide synthase activity in the aorta of young adult (22-week-old) and juvenile (7-week-old) Wistar-Kyoto (WKY) and spontaneously hypertensive rats (SHR). SHR-7: 7-week-old SHR; SHR-22: 22-week-old SHR; WKY-7: 7-week-old WKY rats; WKY22: 22 -week-old WKY rats. Values represent mean \pm SEM of 6-8 rats. Symbols have the following meanings: ${ }^{\mathrm{x}} P<0.05$ compared to age-matched WKY (SHR-7 versus WKY-7, SHR-22 versus WKY22 ), ${ }^{*} P<0.05$ compared to juvenile rats (WKY-22 versus WKY-7, SHR-22 versus SHR-7).

Bonferroni adjustment. To assess depression present at high concentration of ACh-cumulative concentration response curves, the maximal response, and the response at higher ACh concentration at a particular response curve was compared with Dunnett's test. Means were considered to differ significantly when $P<0.05$.

\section{Results}

Systolic blood pressure, heart rate, body weight, LVM/BW ratio, and vascular parameters of experimental groups are shown in Table 1. SBP, heart rate, and LVW/BW were significantly increased and BW and normalized inner diameter of the femoral artery was significantly decreased in both SHR groups as compared to age-matched WKY (Table 1). LVW/BW was lower in all young adult (22-week-old) groups than in juvenile rats. A significant age-dependent increase of BW and normalized inner diameter of the femoral artery was found in 22-week-old WKY and SHR; however age-related SBP increase was present only in 22-week-old SHR compared to juvenile 7-week-old SHR.

NOS activity was increased in SHR compared to agematched WKY (Figure 1). Additionally, there were significant age-related decreases in NOS activity in the aorta of WKY and SHR (Figure 1).

$\mathrm{ACh}(1 \mathrm{nmol} / \mathrm{L}-10 \mu \mathrm{mol} / \mathrm{L})$ and $\mathrm{SNP} \quad(1 \mathrm{nmol} / \mathrm{L}-$ $10 \mu \mathrm{mol} / \mathrm{L}$ ) relaxed the femoral artery from both WKY and SHR in a concentration-dependent manner (Figure 2). 
TABLE 1: Age-related effect on basic biometric and vascular parameters of the femoral artery of juvenile and young adult Wistar-Kyoto (WKY) and spontaneously hypertensive rats (SHR).

\begin{tabular}{|c|c|c|c|c|}
\hline & \multicolumn{2}{|c|}{ WKY } & \multicolumn{2}{|c|}{ SHR } \\
\hline & 7 weeks & 22 weeks & 7 weeks & 22 weeks \\
\hline BW (g) & $168 \pm 8$ & $416 \pm 12^{*}$ & $135 \pm 4^{\mathrm{x}}$ & $382 \pm 8^{* \mathrm{x}}$ \\
\hline $\mathrm{BP}(\mathrm{mmHg})$ & $114 \pm 2$ & $106 \pm 2$ & $159 \pm 4^{\mathrm{x}}$ & $191 \pm 3^{* \mathrm{x}}$ \\
\hline $\mathrm{HR}(\mathrm{bpm})$ & $419 \pm 13$ & $405 \pm 14$ & $545 \pm 10^{\mathrm{x}}$ & $521 \pm 19^{x}$ \\
\hline LVW/BW (mg/g) & $1.99 \pm 0.03$ & $1.44 \pm 0.03^{*}$ & $2.35 \pm 0.06^{\mathrm{x}}$ & $2.18 \pm 0.05^{* \mathrm{x}}$ \\
\hline $\mathrm{ND}(\mu \mathrm{m})$ & $649 \pm 17$ & $769 \pm 12^{*}$ & $556 \pm 20^{\mathrm{x}}$ & $690 \pm 13^{* x}$ \\
\hline $\operatorname{Ser}(\mathrm{mN} / \mathrm{mm})$ & $5.63 \pm 0.25$ & $7.41 \pm 0.15^{*}$ & $5.90 \pm 0.40$ & $6.05 \pm 0.50^{\mathrm{x}}$ \\
\hline $\operatorname{Ser}_{\text {L-NAME }}(\mathrm{mN} / \mathrm{mm})$ & $7.03 \pm 0.45^{\dagger}$ & $8.94 \pm 0.31^{* \dagger}$ & $7.44 \pm 0.38^{\dagger}$ & $7.80 \pm 0.47^{\dagger}$ \\
\hline$E_{\max A C h}(\mathrm{mN} / \mathrm{mm})$ & $4.50 \pm 0.21$ & $4.80 \pm 0.15$ & $3.40 \pm 0.25^{\mathrm{x}}$ & $2.83 \pm 0.19^{\mathrm{x}}$ \\
\hline$E_{\operatorname{max~L-NAME}}(\mathrm{mN} / \mathrm{mm})$ & $3.38 \pm 0.25^{\dagger}$ & $4.54 \pm 0.36^{*}$ & $1.57 \pm 0.20^{\mathrm{x} \dagger}$ & $1.99 \pm 0.40^{\mathrm{x} \dagger}$ \\
\hline$E_{\operatorname{max~SNP}}(\%)$ & $95.3 \pm 0.9$ & $93.9 \pm 1.0$ & $98.6 \pm 0.8$ & $94.0 \pm 0.8$ \\
\hline KPSS (mN/mm) & $6.54 \pm 0.61$ & $9.17 \pm 0.90^{*}$ & $7.42 \pm 0.63$ & $9.78 \pm 0.48^{*}$ \\
\hline
\end{tabular}

ACh: acetylcholine; BW: body weight; BP: blood pressure; $E_{\max \text { ACh }}$ : maximal acetylcholine-induced relaxation based on individual concentration-response curves; $E_{\max \text { L-NAME }}$ : maximal acetylcholine-induced relaxation after L-NAME based on individual concentration-response curves; $E_{\max } \mathrm{SNp}$ : maximal sodium nitroprusside-induced relaxation based on individual concentration-response curves; HR: heart rate; KPSS: high-potassium physiological salt solution; LNAME: $\mathrm{N}^{\mathrm{G}}$-nitro-L-arginine methyl ester; LVW: left heart ventricle weight; ND: normalized diameter of the femoral artery at $13.3 \mathrm{kPa}$ calculated within the normalization procedure; Ser: vascular constriction induced by serotonin $(1 \mu \mathrm{mol} / \mathrm{L}) ; \operatorname{Ser}_{\mathrm{L}-\mathrm{NAME}}$ : vascular constriction induced by serotonin $(1 \mu \mathrm{mol} / \mathrm{L})$ after L-NAME; SNP: sodium nitroprusside. Results are mean \pm S.E.M. of $6-10$ rats. ${ }^{\mathrm{x}} P<0.05$ compared to age-matched WKY (SHR-7 versus WKY-7, SHR-22 versus WKY-22), ${ }^{*} P<0.05$ compared to juvenile rats (WKY-22 versus WKY-7, SHR-22 versus SHR-7), and ${ }^{\dagger} P<0.05$ compared to the respective value before L-NAME.

Application of L-NAME abolished partially the effect of $\mathrm{ACh}$, as illustrated in Figure 3. Since serotonin $(1 \mu \mathrm{mol} / \mathrm{L})$ induced different responses in 7-compared to 22-week-old WKY arteries and also in 22-week-old WKY compared to SHR femoral arteries resulting in a different prerelaxation active tension level (Table 1), the relaxation results were quantitatively expressed as $\mathrm{mN} / \mathrm{mm}$. However, calculations for relaxation responses in percentages (the percent of relaxation calculated relative to the steady-state contraction to Ser) revealed similar differences between the experimental groups and the results correlated well with the extent of prerelaxation active tone (data not shown). AChinduced concentration-response curves were comparable in juvenile and young adult rats (Figure 2(a)). ACh-induced vasorelaxation was lower in SHR than that in age-matched WKY (Figure 2(a)). In SHR, maximal relaxation was achieved at ACh concentration of $3 \cdot 10^{-7} \mathrm{~mol} / \mathrm{L}$ and a slight contractile effect counteracted the relaxant response at higher concentrations of $\mathrm{ACh}$, resulting in a significant decrease in relaxation response at $10^{-5} \mathrm{~mol} / \mathrm{L} \mathrm{ACh}$ compared to the maximum relaxation response (Figure 2(a)). Cumulative addition of the NO donor sodium nitroprusside (SNP) produced similar relaxation responses in the femoral arteries from all experimental groups (Figure 2(b)).

L-NAME attenuated ACh-induced vasorelaxation in all groups investigated (Figure 3 ). The effect of L-NAME on ACh-induced relaxation did not differ between 7-week-old and 22-week-old SHR as compared to age-matched WKY (Figures 3 and 5). However, L-NAME attenuated AChinduced relaxation in a smaller degree in young adult 22week-old rats in both strains as compared to juvenile 7-weekold rats (Figures 3 and 5). When the arteries were pretreated with L-NAME, the femoral arteries from 7- and 22-week-old
SHR rats responded by smaller relaxation to ACh than did the age-matched normotensive WKY arteries (Figures 4(c) and $4(\mathrm{~d})$ ), yet the femoral arteries from young adult 22-week-old rats responded in an even higher extent than did arteries from juvenile 7-week-old rats (Figures 4(a) and 4(b)).

NA-induced responses were biphasic: a transient contraction (early response, phasic contraction), which occurred within the first $10-15 \mathrm{sec}$ and returned nearly to baseline was followed by sustained contraction (delayed response, tonic contraction), which reached steady maximum levels at 5 to $20 \mathrm{~min}$. The tonic response of the 7- and 22-week-old SHR femoral artery to noradrenaline was greater than that in the age-matched WKY (Figure 6(a)). Phasic response was augmented in the 22-week-old SHR compared to the agematched WKY. Aging significantly potentiated the contractions induced by NA in the femoral artery of both SHR and WKY. Unlike the NA-induced contraction, the KCl-induced contraction of the femoral artery seems to be unaffected in hypertensive rats, though an age-related increase was observed both in WKY and SHR (Table 1). Consequently, the ratio between the two contractile agents $(\mathrm{NA} / \mathrm{KCl}$ ) was still higher in the femoral artery of SHR as compared to that of WKY (Figure 6(b)). However only the tonic contraction induced by NA determined in relative values (calculated as percentage of maximal response induced by KPSS) was significantly greater in SHR than in WKY rats.

\section{Discussion}

The possible role of aging affecting vascular responses was studied in relation to experimental hypertension. In this study, we investigated age-related alterations in 


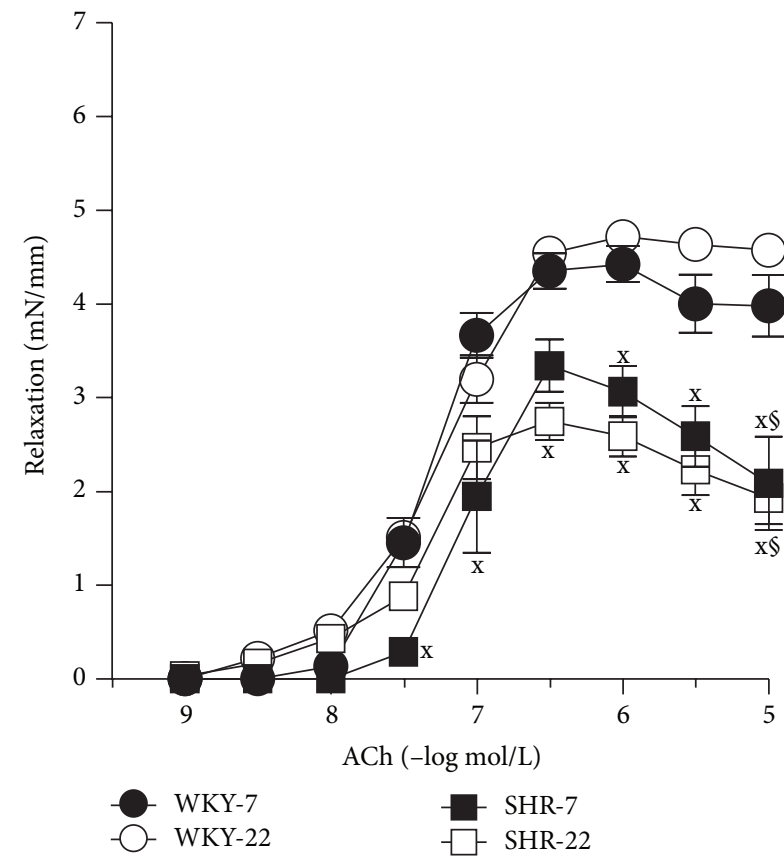

(a)

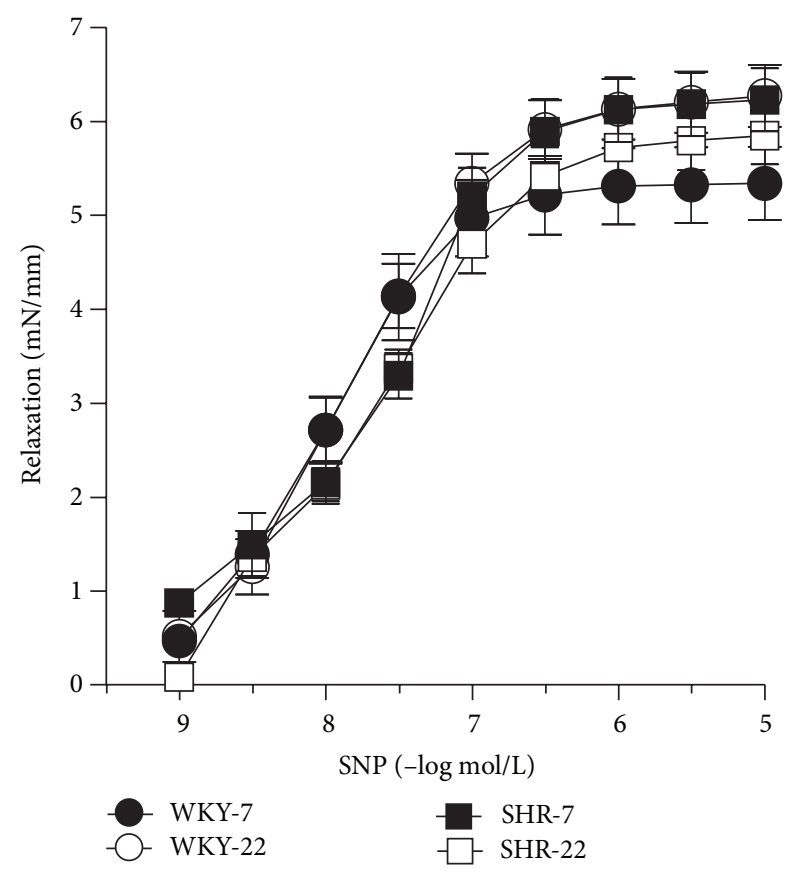

(b)

FIGURE 2: (a) Vascular responses to acetylcholine (ACh) and (b) sodium nitroprusside (SNP) in isolated femoral arteries of 7- and 22-weekold Wistar-Kyoto (WKY) and spontaneously hypertensive rats (SHR). Values represent mean \pm SEM of 6-8 rats. SHR-7: 7-week-old SHR; SHR-22: 22-week-old SHR; WKY-7: 7-week-old WKY rats; WKY-22: 22-week-old WKY rats. Symbols have the following meanings: ${ }^{\mathrm{x}} P$ < 0.05 compared to age-matched WKY (SHR-7 versus WKY-7, SHR-22 versus WKY-22), ${ }^{\S} P<0.05$, compared to maximal relaxation at ACh concentrations $0.3 \mu \mathrm{mol} / \mathrm{L}$ - this significance indicates the release of counterbalancing contracting factors in hypertensive animals.

acetylcholine-induced relaxation of the femoral artery, its L-NAME-sensitive and -resistant components, and total nitric oxide synthase activity in the aortas of juvenile and young adult WKY and SHR. The present study showed that (1) SBP was augmented in 7- and 22-week-old SHR compared to age-matched WKY and age-related augmentation of SBP was seen in SHR but not in WKY; (2) ACh-induced vasorelaxation in the femoral artery in 7-week-old rats did not differ from that in 22-week-old rats in either strain investigated; (3) ACh-induced relaxation in 7- and 22-week-old SHR was attenuated compared with that in age-matched WKY and this endothelial dysfunction originated from reduced NOindependent mechanisms and/or elevated release of EDCF, yet not from NO deficiency; (4) ACh-induced relaxation mediated by NO and NOS activity were attenuated in young adult (22-week-old) WKY and SHR compared with that in juvenile (7-week-old) rats; (5) NA-induced vasoconstriction was augmented in 7- and 22-week-old SHR compared to age-matched WKY; and finally, (6) aging augmented the responses to NA in both WKY and SHR.

In this study we determined NO synthase activity in the aorta and the L-NAME-sensitive component of AChinduced relaxation in the femoral artery as measures of $\mathrm{NO}$ production and bioavailability in the vasculature. Although we could not measure NOS activity in the femoral arteries (due to insufficient amount of tissue for this method), it is assumed that, despite anatomic heterogeneity of the aorta and femoral artery [31], changes in NOS activity in the aorta correspond to those in NO-dependent relaxation of the femoral artery, as this association was shown previously in rats with blunted NO production [37]. Additionally, it is well known that acetylcholine-induced endothelium-dependent relaxation involves besides $\mathrm{NO}$ also other endotheliumderived relaxing factors. We therefore investigated vascular reactivity also in the presence of the nonspecific NOS inhibitor L-NAME, in order to assess NO-dependent (i.e., L-NAME-sensitive) and NO-independent (i.e., L-NAMEresistant) relaxation. Thus changes in NO production along with changes in the $\mathrm{NO}$-dependent component of $\mathrm{ACh}$ induced relaxation are clear indicators of NO bioavailability in the given artery.

Using this experimental approach, we observed that aging (in a relatively early period of life) reduced the total activity of NOS in the aorta and NO-dependent vasorelaxation of the femoral artery in both WKY and SHR. These alterations were however not associated with quantitatively blunted endothelium-dependent relaxations, as determined by the ACh test.

Data from the literature suggest that aging alters vascular function. Several studies have indicated that endotheliumdependent relaxations might decline with age. Aging was found to blunt NO-dependent relaxations in the mesenteric artery of male Wistar rats [8], in the aorta of healthy normotensive male and female rats $[13,14]$, in the common carotid artery of normotensive WKY and spontaneously hypertensive male rats [15], and in the coronary arterioles 


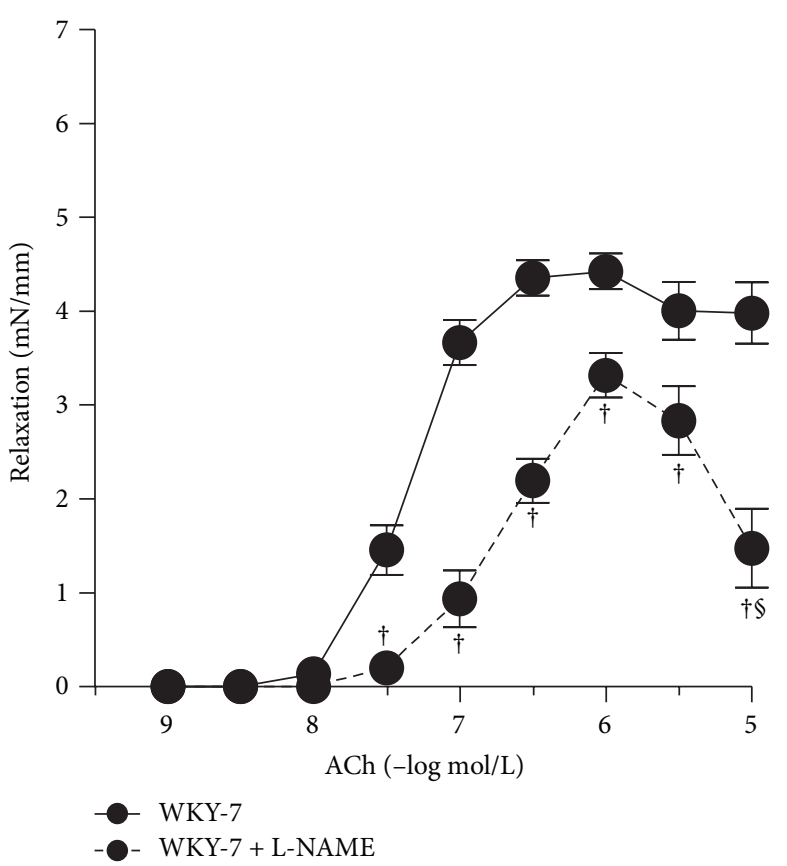

(a)

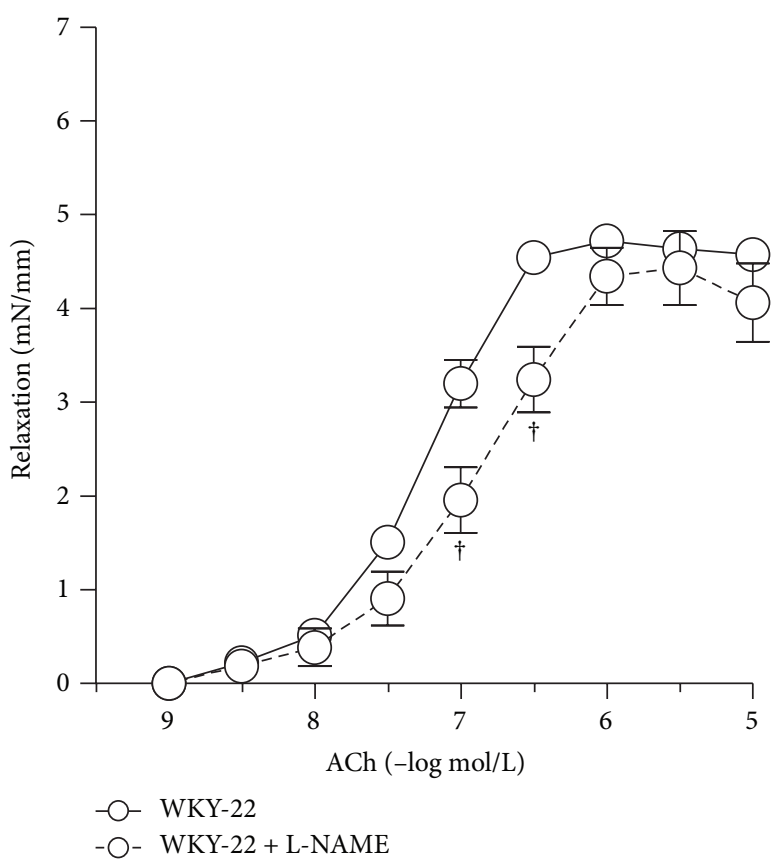

(c)

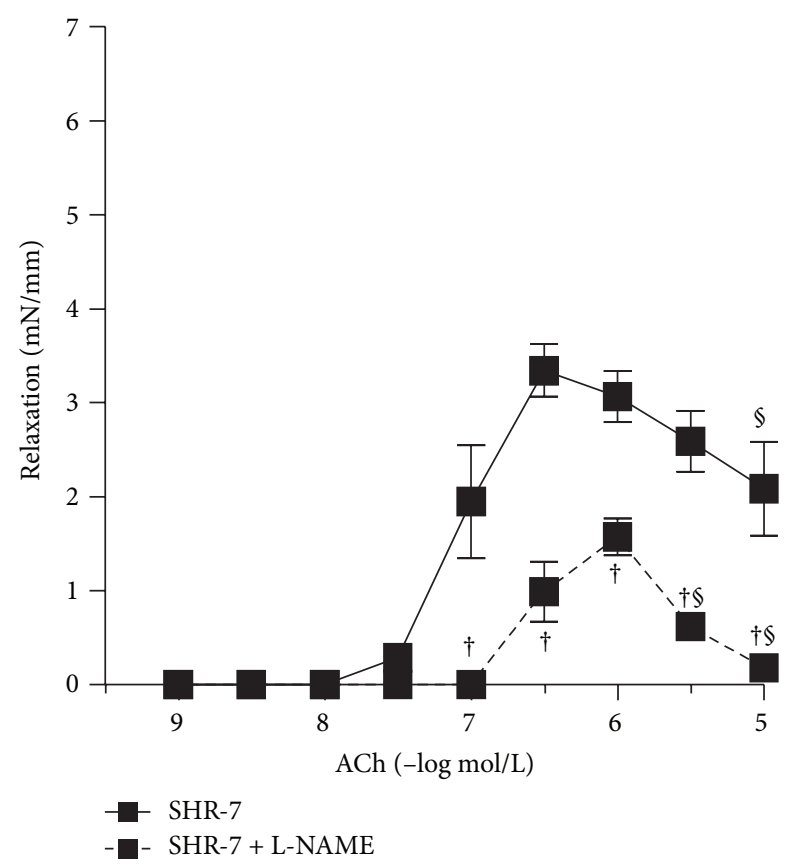

(b)

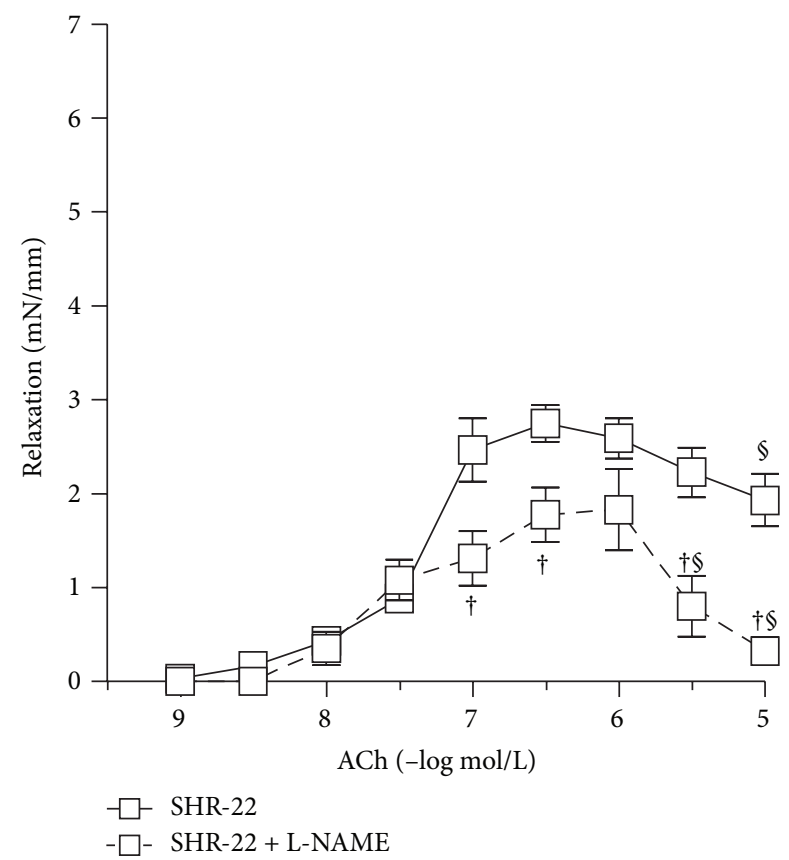

(d)

FIGURE 3: Vascular responses to acetylcholine (ACh) in isolated femoral arteries of 7- and 22-week-old Wistar-Kyoto (WKY) and spontaneously hypertensive rats (SHR) before (full lines) and after (dashed lines) incubation with the nitric oxide (NO) synthase inhibitor $\mathrm{N}^{\mathrm{G}}$ nitro-L-arginine methyl ester (L-NAME, $300 \mu \mathrm{mol} / \mathrm{L}$ ). Endothelium-dependent relaxations in the absence and presence of L-NAME of (a) 7-week-old WKY, (b) 7-week-old SHR, (c) 22-week-old WKY, and (d) 22-week-old SHR. Values represent mean \pm SEM of 6-8 rats. SHR-7: 7-week-old SHR; SHR-22: 22-week-old SHR; WKY-7: 7-week-old WKY rats; WKY-22: 22-week-old WKY rats. Symbols have the following meanings: ${ }^{\dagger} P<0.05$ compared to the respective value before L-NAME; ${ }^{\S} P<0.05$, compared to maximal relaxation at ACh concentrations 0.3 or $1 \mu \mathrm{mol} / \mathrm{L}(\mathrm{a}, \mathrm{c}, \mathrm{d})$-significance indicating release of counterbalancing vasocontractile factors in hypertensive animals and in L-NAMEtreated femoral arteries from juvenile WKY and juvenile and young adult SHR. 


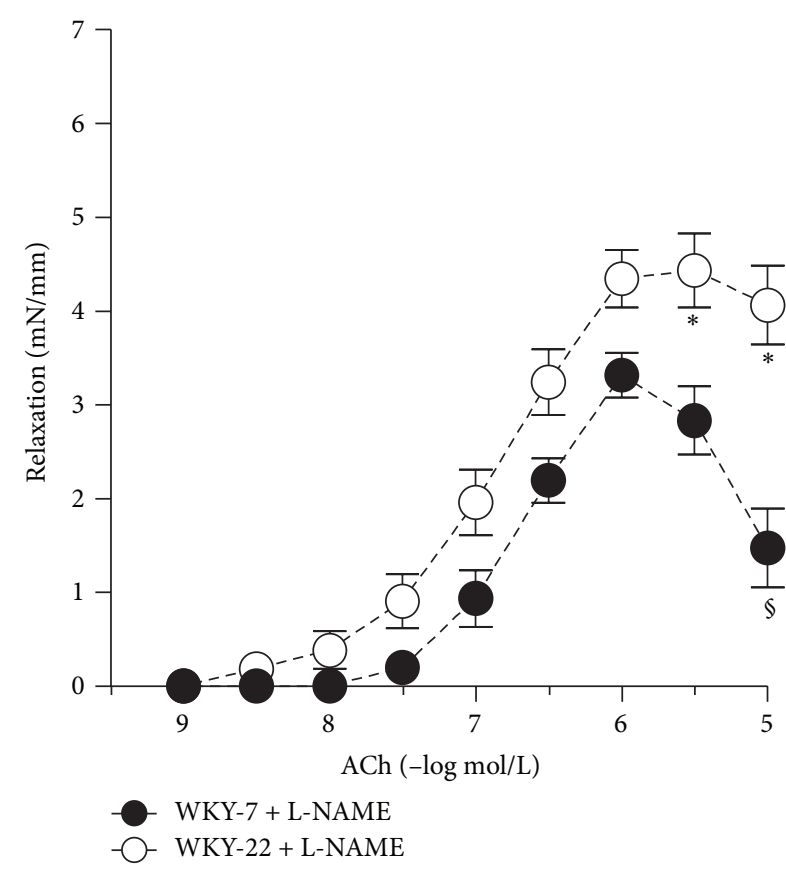

(a)

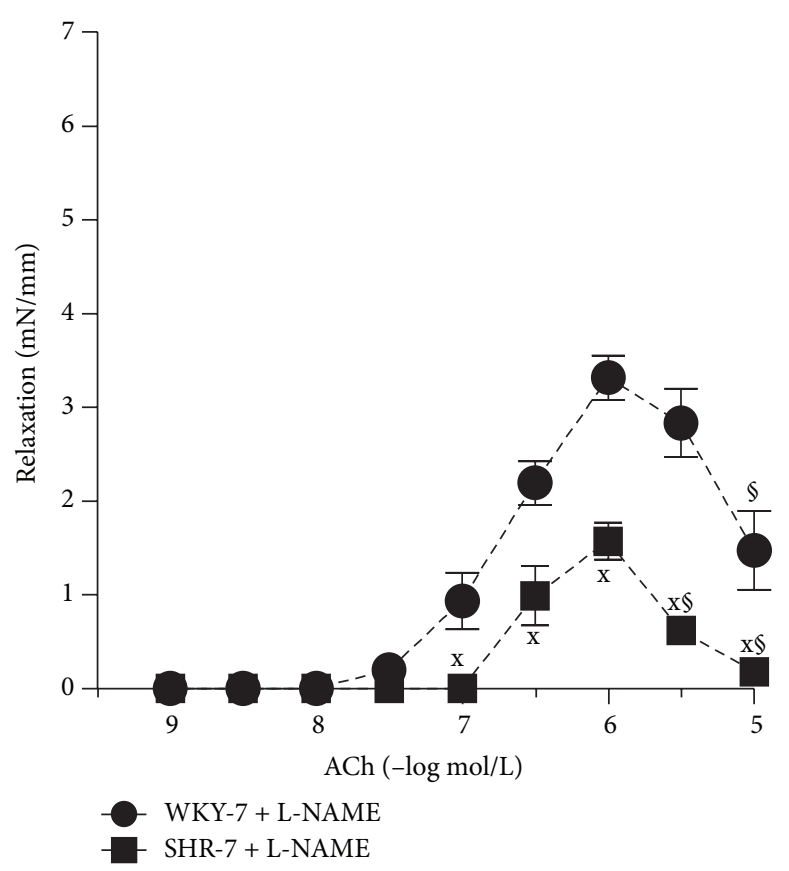

(c)

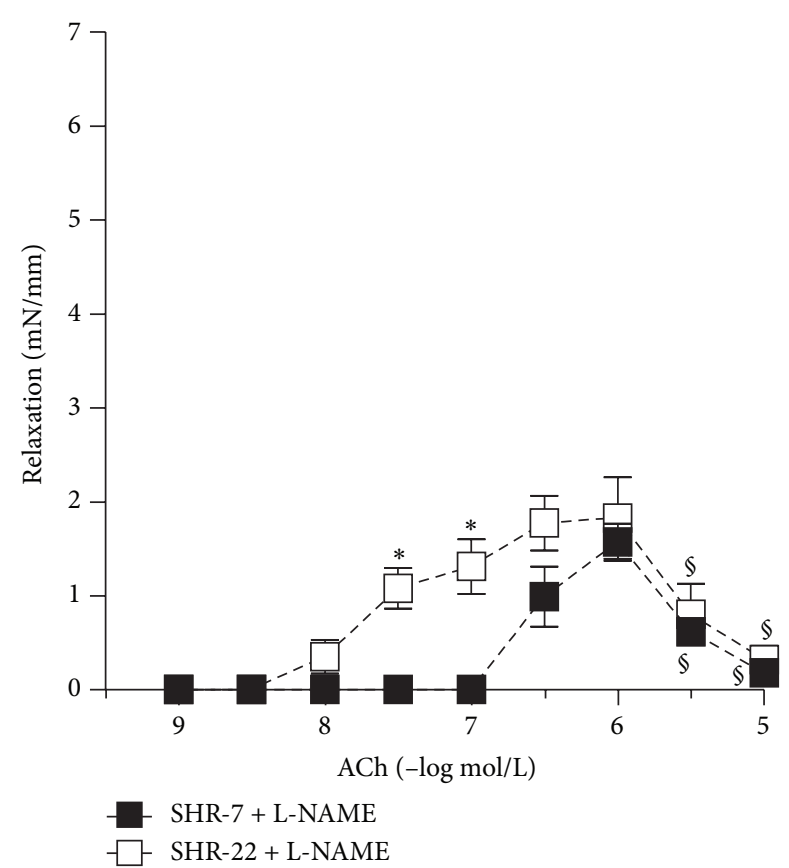

(b)

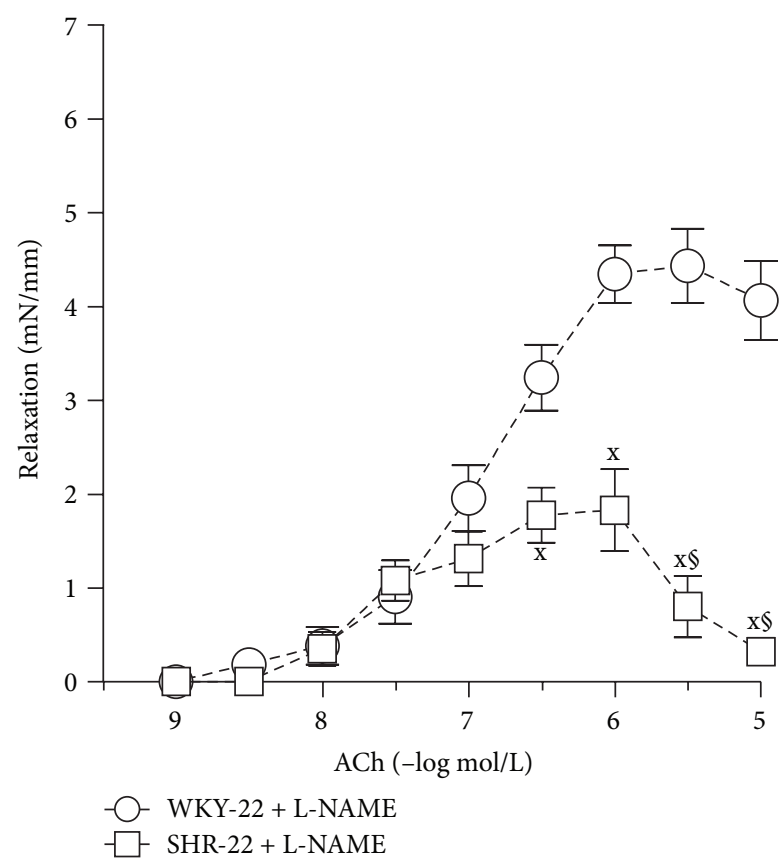

(d)

FIGURE 4: Vascular responses to acetylcholine (ACh) in isolated femoral arteries of 7- and 22-week-old Wistar-Kyoto (WKY) and spontaneously hypertensive rats (SHR) in the presence of nitric oxide $(\mathrm{NO})$ synthase inhibitor $\mathrm{N}^{\mathrm{G}}$-nitro-L-arginine methyl ester (L-NAME, $300 \mu \mathrm{mol} / \mathrm{L}$ ). Age-related effect on endothelium-dependent L-NAME-resistant relaxations in (a) 7- and 22-week-old WKY and (b) 7- and 22-week-old SHR. Differences between normotensive and hypertensive rats in (c) 7-week-old and (d) 22-week-old rats. Values represent mean \pm SEM of 6-8 rats. SHR-7: 7-week-old SHR; SHR-22: 22-week-old SHR; WKY-7: 7-week-old WKY rats; WKY-22: 22-week-old WKY rats. Symbols have the following meanings: ${ }^{\mathrm{x}} \mathrm{P}<0.05$ compared to age-matched WKY (SHR-7 versus WKY-7, SHR-22 versus WKY-22), ${ }^{*} P<0.05$ compared to juvenile rats (WKY-22 versus WKY-7, SHR-22 versus SHR-7), and ${ }^{\S} P<0.05$ compared to maximal relaxation at ACh concentrations 0.3 or $1 \mu \mathrm{mol} / \mathrm{L}$ (a, b, c, d) - significance indicating release of counterbalancing vasocontractile factors in hypertensive animals and in L-NAME-treated femoral arteries from juvenile WKY and juvenile and young adult SHR. 


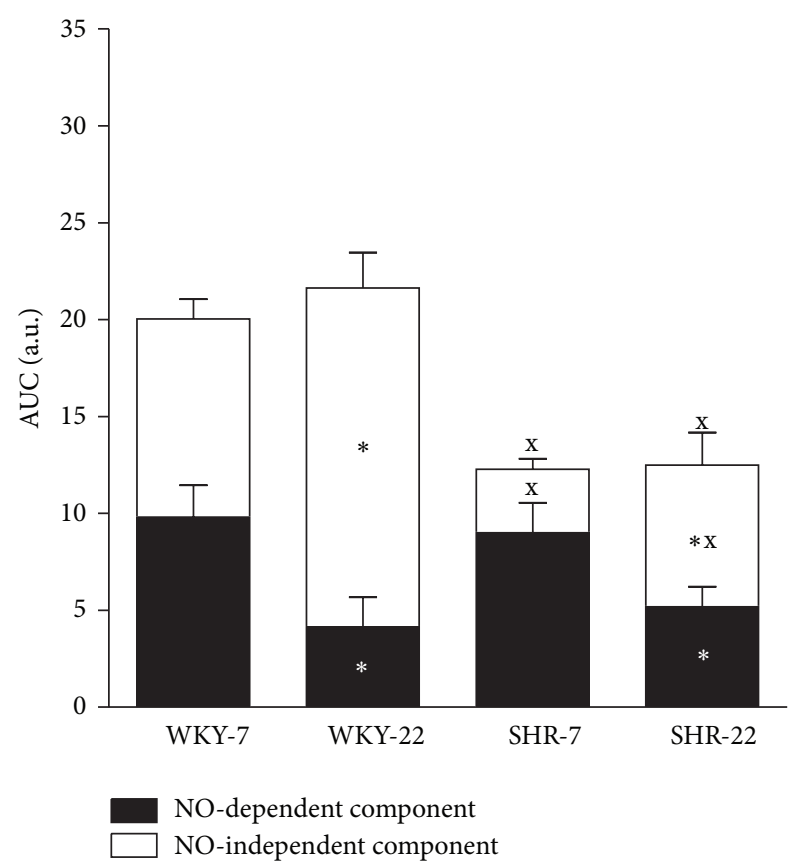

FIGURE 5: Area under the curve (AUC) of the vascular responses, based on the individual concentration-dependent relaxation curves to acetylcholine $(\mathrm{ACh})$ in isolated femoral arteries of 7- and 22week-old Wistar-Kyoto (WKY) and spontaneously hypertensive rats (SHR). Values represent mean \pm SEM of 6-8 rats. SHR-7: 7-weekold SHR; SHR-22: 22-week-old SHR; WKY-7: 7-week-old WKY rats; WKY-22: 22-week-old WKY rats. Marks of significances over the entire columns represent significant differences between the total ACh-induced relaxations. Marks inside the black part indicate significances between NO-dependent component of vasorelaxation and marks inside the white bar represent the significant differences between the NO-independent components. Symbols have the following meanings: ${ }^{\mathrm{x}} P<0.05$ compared to age-matched WKY (SHR7 versus WKY-7, SHR-22 versus WKY-22), ${ }^{*} P<0.05$ compared to juvenile rats (WKY-22 versus WKY-7, SHR-22 versus SHR-7).

from Sprague-Dawley male rats [16], but it did not decrease relaxation in the pulmonary artery of normotensive female rats [14].

In this study, despite unchanged total ACh-induced relaxation, we observed significant qualitative age-related changes in the mechanism of relaxation by acute preincubation with L-NAME. NO-dependent components of the ACh-induced relaxation were reduced in young adult rats in association with reduced NOS activity in both strains investigated. Although we did not measure the activity of individual NOS isoforms, there are studies indicating the participation of eNOS and iNOS in age-related alterations [11]. In contrast, during a period of life comparable to that investigated in our study (4 weeks versus 14-17 weeks), reduction of eNOS activity was not observed in the aortas of WKY and SHR [41]. Chou et al. [41] found reduced activity of eNOS only in the aortas of WKY and that in much older rats (63week-old). In addition, the same study showed that the basal activity and protein expression of iNOS were detected only in elderly (63-week-old) SHR and WKY and in adult (14-to-17-week old) SHR yet not in adult WKY, suggesting that the abnormal expression of iNOS is associated with hypertension. This abnormal iNOS expression might be implicated also in our study concerning the finding that SHR exhibited a significantly increased NOS activity than did agematched WKY rats. Thus the observed increased total NOS activity in the aortas from SHR as compared to WKY at both ages studied may be related to the abnormal expression of iNOS or to a compensatory mechanism by eNOS activated in hypertension $[42,43]$. However, elevated NOS expression need not be always associated with improved enzyme activity. Indeed, in the aortas from aged male Wistar rats, the expression of eNOS isoform was enhanced; however its activity was markedly reduced [44]. Reduced activity of NOS was also found in this study and may account for age-related decrease of NO-mediated vasorelaxation, independently of NO breakdown by reactive oxygen species [5].

Interestingly, we observed a significant age-related increase in the L-NAME-resistant (i.e., NO-independent) component of ACh-induced relaxation in WKY and SHR. This result is in contradistinction to previous reports which showed a rather decreased EDHF-mediated relaxation in vascular aging [8]. The disparity with our results may be related to differences in age and vascular bed of the animals studied. Although it is assumed that elevation of the NOindependent component of vasorelaxation in young adult rats (versus juvenile) was elicited by compensatory release of $\mathrm{EDHF}(\mathrm{s})$ and/or vasodilatory prostanoids, the effect of other endothelium derived factors cannot be ruled out.

Our findings also indicate that other than NO-mediated mechanisms can effectively be activated to compensate the loss of NO bioavailability in early vascular aging. This explanation has been suggested in the study of Sofola et al. [45] who indicated that EDHF might compensate the loss of $\mathrm{NO}$ and preserve the endothelium-dependent relaxation in the mesenteric arteries of hypertensive rats in which hypertension was induced by a high-salt diet. Moreover, compensatory EDHF production has been suggested to occur in normotensive rats after acute NOS inhibition [46]. In addition, the compensatory release of vasodilatory prostanoids may be involved $[5,47]$.

Nevertheless, our study showed that NO-independent endothelial dysfunction was present in SHR even at the age of 7 weeks. Similarly did Mori et al. [36] show that the EDHF-mediated responses were attenuated before the loss of NO-mediated dilatation in the femoral resistance arteries of SHR compared to WKY. Our results also indicated that the effect of L-NAME on vasorelaxation did not differ in WKY and age-matched SHR; thus there were no significant differences in NO-mediated responses. Similarly, in resistance femoral arteries, there were no differences in NO-mediated vasodilatation in 5- and 15-week-old SHR compared to WKY [36]. We also assume that the decrease of NO-independent relaxation of the SHR femoral artery may be related to a reduced EDHF-dependent component because $\mathrm{PGI}_{2}$ inhibition by indomethacin failed to show any effects on ACh-induced relaxation of hindlimb arteries of SHR and WKY at various periods of life [36]. Yet as there is a complex cross-talk among individual EDRFs [10], 


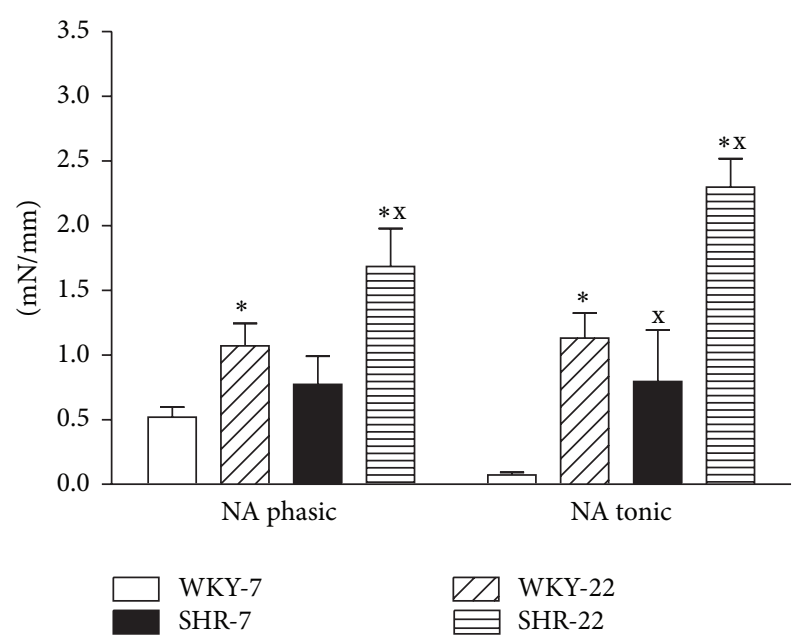

(a)
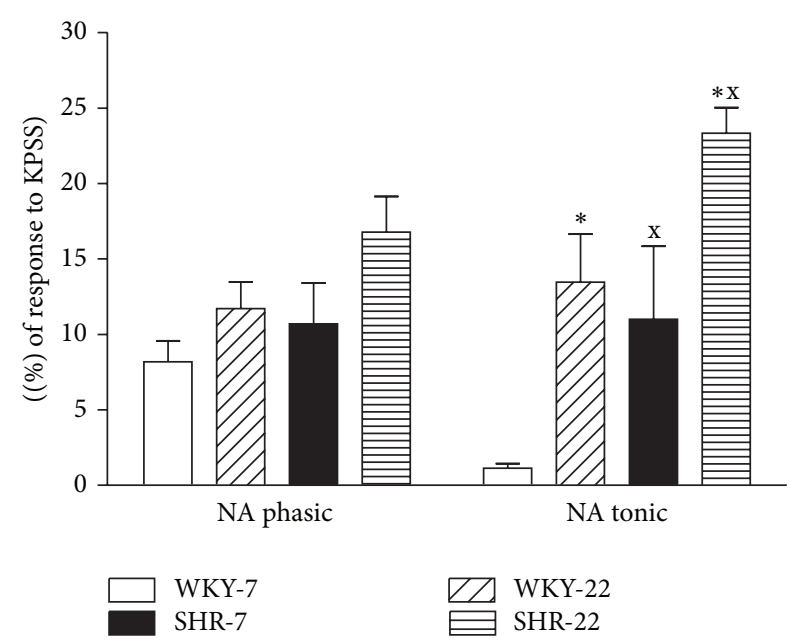

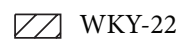

SHR-22

(b)

Figure 6: Vascular constrictions induced by noradrenaline (NA, $10 \mu \mathrm{mol} / \mathrm{L}$ ) in the femoral artery of young adult (22-week-old) and juvenile (7-week-old) Wistar-Kyoto (WKY) and spontaneously hypertensive rats (SHR). SHR-7: 7-week-old SHR; SHR-22: 22-week-old SHR; WKY-7: 7-week-old WKY rats; WKY-22: 22-week-old WKY rats. Values represent mean \pm SEM of 6-8 rats. Symbols have the following meanings: ${ }^{\mathrm{x}} P<0.05$ compared to age-matched WKY (SHR-7 versus WKY-7, SHR-22 versus WKY-22), ${ }^{*} P<0.05$ compared to juvenile rats (WKY-22 versus WKY-7, SHR-22 versus SHR-7).

the contribution of individual EDRFs to alterations of NOindependent components of ACh-induced relaxation needs to be evaluated in specifically designed studies.

Regarding vasoconstriction, elevated responses to serotonin were observed in all groups investigated after NOS inhibition. As the active tone level was decreased (not increased) in young adult SHR and comparable in the arteries from juvenile rats, it is not assumed that the level of precontraction tone could account for the reduced endothelial relaxation in SHR as compared to age-matched WKY. We found an increase in NA-induced vasoconstriction in hypertensive rats compared to normotensive age-matched controls and aging augmented further the noradrenaline-induced contractions of femoral arteries in SHR. This finding is in contrast to the report of Konishi and Su [26] showing unaffected responses to NA in 15-16-week-old SHR femoral arteries. However, other investigators $[22,23]$ reported similar augmentations of NA responses in the femoral artery of 16-20-week-old male SHR compared to age-matched normotensive rats. A similar augmentation of NA-induced responses was found in the aorta of male SHR [48] and the femoral artery of adult female SHR [49]. Since KCl acts directly on smooth muscle cells [25], the augmented NA response observed in SHR, in relation to $\mathrm{KCl}$, indicates alterations in NA receptors and/or signaling, alterations of $\mathrm{Ca}^{2+}$ handling and sensitivity [22, 50-52], and structural remodeling of the arteries. Furthermore, EDCF contribution to NA-induced contraction of femoral arteries was reported to be similarly enhanced in adult (7-month-old) SHR and aged (14-month-old) normotensive WKY rats [39]. In addition, endothelium-dependent contractions elicited by high concentrations of acetylcholine were described both in hypertensive and in aged normotensive rats [53-55]. Cyclooxygenase-dependent EDCF production was found to be characteristic of aging $[5,56]$. Our findings also support the role of $\mathrm{EDCF}(\mathrm{s})$ in hypertension-related endothelial dysfunction in SHR (Figures 3(b) and 3(d)). Moreover, augmented $\mathrm{EDCF}(\mathrm{s})$-induced decline in relaxation responses after L-NAME-pretreatment in both SHR groups and juvenile WKY suggests neutralization of EDCF(s) action by NO. This mechanism was reported previously by Auch-Schwelk et al. [57].

It has been widely accepted that aging and hypertension do not affect sensitivity of vascular smooth muscle to NO (e.g., relaxation induced by endothelium-independent vascular smooth muscle relaxants such as sodium nitroprusside) and yet some conflicting findings were reported $[15,58,59]$. In our experiments, SNP-induced concentration-dependent relaxation did not differ between the groups, indicating that a decrease in the NO-mediated $\mathrm{ACh}$-induced relaxations was due to specific impairment of the endothelium-dependent mechanism rather than to changes in vascular smooth muscle cells. Several studies have suggested that reduced NO bioavailability during aging can be related to increased production of reactive oxygen species. Indeed, aging was associated with blunted endothelium-dependent relaxations and excessive vascular formation of reactive oxygen species in Wistar, SHR, and WKY rats $[8,60]$. It should however be noted that the oxidative stress theory of aging has to be further elucidated due to significant animal-strain-related differences in the effect of reactive oxygen species [61, 62].

It is well known that elevated vascular resistance along with increased stiffness of conduit arteries participate in aging and in hypertension development $[2,63]$. We found that SBP increased significantly with aging in SHR, which was not observed in WKY. Thus chronic exposure of the femoral artery to higher pressure may not be the primary cause 
of age-related decrease of NO synthase inhibitor sensitive response to $\mathrm{ACh}$. Moreover, our results suggest that agerelated elevation of blood pressure between the 7 th and 22nd week of life in SHR does not result from worsened endothelium-dependent relaxation but rather from elevated sympathetic vasoconstriction.

An elevated sympathetic tone in SHR was shown previously $[19,20]$ and our observation of increased heart rate in SHR at both ages studied is in agreement with these studies. Moreover, it has been reported that the hyperactivity of the sympathetic nervous system could induce structural and functional alterations of the heart and blood vessels in SHR [20, 64-66]. In addition, age-related decrease in the $\mathrm{NO}$-dependent relaxation in the femoral artery along with its structural remodeling may be implicated in the pathogenesis of peripheral artery disease. Further studies are however needed to evaluate the underlying factors and the exact mechanism of age-related decrease in NO-dependent vasorelaxation in the femoral artery and their involvement in the pathogenesis of peripheral artery disease and hypertension.

\section{Conclusions}

In our study aging between the 7th and 22nd week of life was associated with decreased vascular NO production and NO-mediated vasorelaxation. However reduction in $\mathrm{NO}$ bioavailability did not result in endothelial dysfunction as the reduction of $\mathrm{NO}$-dependent component of relaxation was fully compensated by accentuation of NO-independent relaxation in both WKY and SHR. The results suggest that NO-independent mechanisms can act as a salvage system to maintain endothelial function in situations associated with decreased NO bioavailability, at least in early periods of life. The exact role of $\mathrm{NO}$ in aging and hypertension remains however still open.

\section{Conflict of Interests}

The authors declare that there is no conflict of interests.

\section{Acknowledgments}

The authors thank Mrs. Jana Petova for her technical assistance and help with housing the animals. This study was supported by the Slovak Grant Agency for Science, Grant no. 2/0084/14, and the Slovak Research and Development Agency, Grant no. APVV-0523-10.

\section{References}

[1] E. G. Lakatta and D. Levy, "Arterial and cardiac aging: major shareholders in cardiovascular disease enterprises. Part I. Aging arteries: a "set up" for vascular disease," Circulation, vol. 107, no. 1, pp. 139-146, 2003.

[2] P. M. Nilsson, P. Boutouyrie, and S. Laurent, "Vascular aging: a tale of EVA and ADAM in cardiovascular risk assessment and prevention," Hypertension, vol. 54, no. 1, pp. 3-10, 2009.

[3] K. Egashira, T. Inou, Y. Hirooka et al., "Effects of age on endothelium-dependent vasodilation of resistance coronary artery by acetylcholine in humans," Circulation, vol. 88 , no. 1, pp. 7781, 1993.

[4] D. S. Celermajer, K. E. Sorensen, D. J. Spiegelhalter, D. Georgakopoulos, J. Robinson, and J. E. Deanfield, "Aging is associated with endothelial dysfunction in healthy men years before the age-related decline in women," Journal of the American College of Cardiology, vol. 24, no. 2, pp. 471-476, 1994.

[5] R. L. Matz, M. A. de Sotomayor, C. Schott, J. C. Stoclet, and R. Andriantsitohaina, "Vascular bed heterogeneity in age-related endothelial dysfunction with respect to NO and eicosanoids," British Journal of Pharmacology, vol. 131, no. 2, pp. 303-311, 2000.

[6] R. L. Matz, C. Schott, J. C. Stoclet, and R. Andriantsitohaina, "Age-related endothelial dysfunction with respect to nitric oxide, endothelium-derived hyperpolarizing factor and cyclooxygenase products," Physiological Research, vol. 49, no. 1, pp. 11-18, 2000.

[7] S. Taddei, A. Virdis, L. Ghiadoni et al., "Age-related reduction of NO availability and oxidative stress in humans," Hypertension, vol. 38, no. 2, pp. 274-279, 2001.

[8] S. Dal-Ros, C. Bronner, C. Auger, and V. B. Schini-Kerth, "Red wine polyphenols improve an established aging-related endothelial dysfunction in the mesenteric artery of middleaged rats: role of oxidative stress," Biochemical and Biophysical Research Communications, vol. 419, no. 2, pp. 381-387, 2012.

[9] M. Feletou and P. M. Vanhoutte, "Endothelial dysfunction: a multifaceted disorder (The Wiggers Award Lecture)," The American Journal of Physiology-Heart and Circulatory Physiology, vol. 291, pp. 985-1002, 2006.

[10] I. Bernatova, "Endothelial dysfunction in experimental models of arterial hypertension: cause or consequence?" BioMed Research International, vol. 2014, Article ID 598271, 14 pages, 2014.

[11] H. J. Yoon, S. W. Cho, B. W. Ahn, and S. Y. Yang, "Alterations in the activity and expression of endothelial NO synthase in aged human endothelial cells," Mechanisms of Ageing and Development, vol. 131, no. 2, pp. 119-123, 2010.

[12] A. M. Briones, M. Salaices, and E. Vila, "Ageing alters the production of nitric oxide and prostanoids after IL- $1 \beta$ exposure in mesenteric resistance arteries," Mechanisms of Ageing and Development, vol. 126, no. 6-7, pp. 710-721, 2005.

[13] B. van der Loo, R. Labugger, J. N. Skepper et al., "Enhanced peroxynitrite formation is associated with vascular aging," Journal of Experimental Medicine, vol. 192, no. 12, pp. 1731-1743, 2000.

[14] M. R. Tschudi, M. Barton, N. A. Bersinger et al., "Effect of age on kinetics of nitric oxide release in rat aorta and pulmonary artery," Journal of Clinical Investigation, vol. 98, no. 4, pp. 899905, 1996.

[15] K. Hongo, T. Nakagomi, N. F. Kassell et al., "Effects of aging and hypertension on endothelium-dependent vascular relaxation in rat carotid artery," Stroke, vol. 19, no. 7, pp. 892-897, 1988.

[16] A. Csiszar, Z. Ungvari, J. G. Edwards et al., "Aging-induced phenotypic changes and oxidative stress impair coronary arteriolar function," Circulation Research, vol. 90, no. 11, pp. 1159-1166, 2002.

[17] Y. Yamori and K. Okamoto, "Spontaneous hypertension in rats versus essential hypertension in man," Singapore Medical Journal, vol. 14, no. 3, pp. 393-394, 1973.

[18] M. Pintérová, J. Kuneš, and J. Zicha, "Altered neural and vascular mechanisms in hypertension," Physiological Research, vol. 60 , no. 3, pp. 381-402, 2011. 
[19] J. Zicha and J. Kuneš, "Ontogenetic aspects of hypertension development: analysis in the rat," Physiological Reviews, vol. 79, no. 4, pp. 1227-1282, 1999.

[20] M. Behuliak, M. Pintérová, J. Kuneš, and J. Zicha, "Vasodilator efficiency of endogenous prostanoids, $\mathrm{Ca}^{2+}$-activated $\mathrm{K}^{+}$channels and nitric oxide in rats with spontaneous, salt-dependent or NO-deficient hypertension," Hypertension Research, vol. 34, no. 8, pp. 968-975, 2011.

[21] A. Zemančíková and J. Török, "Effect of chronic nifedipine treatment on blood pressure and adrenergic responses of isolated mesenteric artery in young rats with developing spontaneous hypertension," Physiological Research, vol. 58, no. 6, pp. 921-925, 2009.

[22] S. Líšková, M. Petrová, P. Karen, J. Kuneš, and J. Zicha, "Influence of calcium-dependent potassium channel blockade and nitric oxide inhibition on norepinephrine-induced contractions in two forms of genetic hypertension," Journal of the American Society of Hypertension, vol. 4, no. 3, pp. 128-134, 2010.

[23] M. Petrova, S. Liskova, R. Vojtko et al., "Vascular reactivity of arteria femoralis in adult and aged spontaneously hypertensive and Wistar-Kyoto rats," Bratislavske lekarske listy, vol. 114, pp. 553-555, 2013.

[24] L. Paulis, S. Lišková, M. Pintérová, Z. Dobešová, J. Kuneš, and J. Zicha, "Nifedipine-sensitive noradrenergic vasoconstriction is enhanced in spontaneously hypertensive rats: the influence of chronic captopril treatment," Acta Physiologica, vol. 191, no. 4, pp. 255-266, 2007.

[25] A. Puzserova, J. Kopincova, P. Slezak, P. Balis, and I. Bernatova, "Endothelial dysfunction in femoral artery of the hypertensive rats is nitric oxide independent," Physiological Research, vol. 62, pp. 615-629, 2013.

[26] M. Konishi and C. Su, "Role of endothelium in dilator responses of spontaneously hypertensive rat arteries," Hypertension, vol. 5, no. 6, pp. 881-886, 1983.

[27] H. Suzuki and Y. Iso, "Clinical application of vascular regenerative therapy for peripheral artery disease," BioMed Research International, vol. 2013, Article ID 179730, 6 pages, 2013.

[28] M. A. Delbin, A. S. Silva, E. Antunes, and A. Zanesco, "Interaction between serotoninergic-and $\beta$-adrenergic receptors signaling pathways in rat femoral artery," Arquivos Brasileiros de Cardiologia, vol. 98, no. 1, pp. 29-34, 2012.

[29] M. M. Joosten, J. K. Pai, M. L. Bertoia et al., "Associations between conventional cardiovascular risk factors and risk of peripheral artery disease in men," Journal of the American Medical Association, vol. 308, pp. 1660-1667, 2012.

[30] C. Diehm, S. Kareem, and H. Lawall, "Epidemiology of peripheral arterial disease," Journal of Vascular Diseases, vol. 33, no. 4, pp. 183-189, 2004.

[31] M. Barton, F. Cosentino, R. P. Brandes, P. Moreau, S. Shaw, and T. F. Lüscher, "Anatomic heterogeneity of vascular aging: role of nitric oxide and endothelin," Hypertension, vol. 30, no. 4, pp. 817-824, 1997.

[32] A. Puzserova, P. Slezak, P. Balis, and I. Bernatova, "Long-term social stress induces nitric oxide-independent endothelial dysfunction in normotensive rats," Stress, vol. 16, pp. 331-339, 2013.

[33] M. J. Mulvany and W. Halpern, "Contractile properties of small arterial resistance vessels in spontaneously hypertensive and normotensive rats," Circulation Research, vol. 41, no. 1, pp. 1926, 1977.

[34] S. Fujimoto, K. S. Fujimoto, and A. Moriyama, "Diminished $\beta$-adrenoceptor-mediated relaxation of femoral arteries from young spontaneously hypertensive rats," Autonomic Neuroscience, vol. 87, no. 2-3, pp. 178-186, 2001.

[35] A. Puzserova and I. Bernatova, "Chronic social stress increases nitric oxide-dependent vasorelaxation in normotensive rats," Interdisciplinary Toxicology, vol. 3, no. 4, pp. 109-117, 2010.

[36] Y. Mori, M. Ohyanagi, S. Koida, A. Ueda, K. Ishiko, and T. Iwasaki, "Effects of endothelium-derived hyperpolariziing factor and nitric oxide on endothelial function in femoral resistance arteries of spontaneously hypertensive rats," Hypertension Research, vol. 29, no. 3, pp. 187-195, 2006.

[37] L. Paulis, J. Zicha, J. Kunes et al., "Regression of L-NAMEinduced hypertension: the role of nitric oxide and endotheliumderived constricting factor," Hypertension Research, vol. 31, no. 4, pp. 793-803, 2008.

[38] K. Hansen and O. A. Nedergaard, "Methodologic aspects of acetylcholine-evoked relaxation of rabbit aorta," Journal of Pharmacological and Toxicological Methods, vol. 41, no. 4, pp. 153-159, 1999.

[39] S. Líšková, M. Petrová, P. Karen, J. Kuneš, and J. Zicha, "Effects of aging and hypertension on the participation of endotheliumderived constricting factor (EDCF) in norepinephrine-induced contraction of rat femoral artery," European Journal of Pharmacology, vol. 667, no. 1-3, pp. 265-270, 2011.

[40] O. H. Lowry, N. J. Rosebrough, A. L. Farr, and R. J. Randall, "Protein measurement with the Folin phenol reagent," The Journal of biological chemistry, vol. 193, no. 1, pp. 265-275, 1951.

[41] T. C. Chou, M. H. Yen, C. Y. Li, and Y. A. Ding, "Alterations of nitric oxide synthase expression with aging and hypertension in rats," Hypertension, vol. 31, no. 2, pp. 643-648, 1998.

[42] O. Pechanova, J. Zicha, S. Kojsova, Z. Dobesova, L. Jendekova, and J. Kunes, "Effect of chronic N-acetylcysteine treatment on the development of spontaneous hypertension," Clinical Science, vol. 110, pp. 235-242, 2006.

[43] J. Török, "Participation of nitric oxide in different models of experimental hypertension," Physiological Research, vol. 57, no. 6, pp. 813-825, 2008.

[44] M. R. Cernadas, L. S. de Miguel, M. García-Durán et al., "Expression of constitutive and inducible nitric oxide synthases in the vascular wall of young and aging rats," Circulation Research, vol. 83, no. 3, pp. 279-286, 1998.

[45] O. A. Sofola, A. Knill, R. Hainsworth, and M. Drinkhill, "Change in endothelial function in mesenteric arteries of Sprague-Dawley rats fed a high salt diet," Journal of Physiology, vol. 543, no. 1, pp. 255-260, 2002.

[46] J. Bauersachs, R. Popp, M. Hecker, E. Sauer, I. Fleming, and R. Busse, "Nitric oxide attenuates the release of endotheliumderived hyperpolarizing factor," Circulation, vol. 94, no. 12, pp. 3341-3347, 1996.

[47] D. Henrion, E. Dechaux, F. J. Dowell et al., "Alteration of flowinduced dilatation in mesenteric resistance arteries of L-NAME treated rats and its partial association with induction of cyclooxygenase-2," British Journal of Pharmacology, vol. 121, no. 1, pp. 83-90, 1997.

[48] S. Arribas, J. Marin, A. Ponte, G. Balfagon, and M. Salaices, "Norepinephrine-induced relaxations in rat aorta mediated by endothelial beta adrenoceptors. Impairment by ageing and hypertension," Journal of Pharmacology and Experimental Therapeutics, vol. 270, no. 2, pp. 520-527, 1994.

[49] M. Asano, K. Aoki, and T. Matsuda, "Reduced beta adrenoreceptor interactions of norepinephrine enhance contraction in the femoral artery from spontaneously hypertensive rats," 
Journal of Pharmacology and Experimental Therapeutics, vol. 223, no. 1, pp. 207-214, 1982.

[50] G. J. Lagaud, V. Randriamboavonjy, G. Roul, J. C. Stoclet, and R. Andriantsitohaina, "Mechanism of $\mathrm{Ca}^{2+}$ release and entry during contraction elicited by norepinephrine in rat resistance arteries," The American Journal of Physiology-Heart and Circulatory Physiology, vol. 276, no. 1, pp. H300-H308, 1999.

[51] F. P. Field and E. E. Soltis, "Vascular reactivity in the spontaneously hypertensive rat: effect of high pressure stress and extracellular calcium," Hypertension, vol. 7, no. 2, pp. 228-235, 1985.

[52] M. S. Bal, L. Paulis, J. Zicha, and J. Kuneš, "Effect of protein kinase $\mathrm{C}$ and protein kinase $\mathrm{A}$ inhibitors on contraction of isolated femoral arteries of SHR and Wistar rats," Physiological Research, vol. 58, no. 6, pp. 793-798, 2009.

[53] P. M. Vanhoutte, "Endothelial dysfunction-The first step toward coronary arteriosclerosis," Circulation Journal, vol. 73, no. 4, pp. 595-601, 2009.

[54] M. Félétou, T. J. Verbeuren, and P. M. Vanhoutte, "Endothelium-dependent contractions in SHR: a tale of prostanoid TP and IP receptors," British Journal of Pharmacology, vol. 156, no. 4, pp. 563-574, 2009.

[55] M. S. K. Wong and P. M. Vanhoutte, "COX-mediated endothelium-dependent contractions: from the past to recent discoveries," Acta Pharmacologica Sinica, vol. 31, no. 9, pp. 1095$1102,2010$.

[56] D. Versari, E. Daghini, A. Virdis, L. Ghiadoni, and S. Taddei, "Endothelium-dependent contractions and endothelial dysfunction in human hypertension," British Journal of Pharmacology, vol. 157, no. 4, pp. 527-536, 2009.

[57] W. Auch-Schwelk, Z. S. Katusic, and P. M. Vanhoutte, "Nitric oxide inactivates endothelium-derived contracting factor in the rat aorta," Hypertension, vol. 19, no. 5, pp. 442-445, 1992.

[58] H. Wuorela, P. Arvola, M. Kahonen, H. Vapaatalo, and I. Porsti, "Arterial smooth muscle responses in adult and moderately aged spontaneously hypertensive rats," Pharmacology and Toxicology, vol. 74, no. 3, pp. 167-173, 1994.

[59] S. Fujimoto, Y. Dohi, K. Aoki, M. Asano, and T. Matsuda, "Diminished $\beta$-adrenoceptor-mediated relaxation of arteries from spontaneously hypertensive rats before and during development of hypertension," European Journal of Pharmacology, vol. 136, no. 2, pp. 179-187, 1987.

[60] S. Simão, P. Gomes, V. Pinto et al., "Age-related changes in renal expression of oxidant and antioxidant enzymes and oxidative stress markers in male SHR and WKY rats," Experimental Gerontology, vol. 46, no. 6, pp. 468-474, 2011.

[61] R. Buffenstein, Y. H. Edrey, T. Yang, and J. Mele, "The oxidative stress theory of aging: embattled or invincible? Insights from non-traditional model organisms," Age, vol. 30, no. 2-3, pp. 99109,2008

[62] V. I. Pérez, A. Bokov, H. V. Remmen et al., "Is the oxidative stress theory of aging dead?" Biochimica et Biophysica Acta, vol. 1790, no. 10, pp. 1005-1014, 2009.

[63] S. M. Arribas, A. M. Briones, C. Bellingham et al., "Heightened aberrant deposition of hard-wearing elastin in conduit arteries of prehypertensive SHR is associated with increased stiffness and inward remodeling," The American Journal of PhysiologyHeart and Circulatory Physiology, vol. 295, no. 6, pp. H2299H2307, 2008.

[64] M. Cebova and F. Kristek, "Age-dependent ultrastructural changes of coronary artery in spontaneously hypertensive rats,"
General Physiology and Biophysics, vol. 30, no. 4, pp. 364-372, 2011.

[65] M. Gerová and F. Kristek, "Integrative pattern of vasomotor efficiency in SHR during ontogenesis," Physiological Research, vol. 57, no. 5, pp. 677-684, 2008.

[66] A. Zemancíková and J. Török, "Diminished contractile responses of isolated conduit arteries in two rat models of hypertension," The Chinese Journal of Physiology, vol. 56, no. 4, pp. 230-235, 2013. 


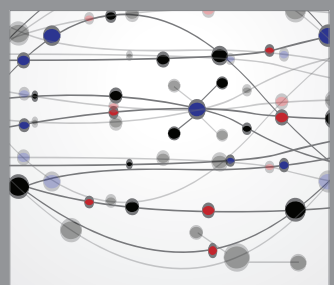

The Scientific World Journal
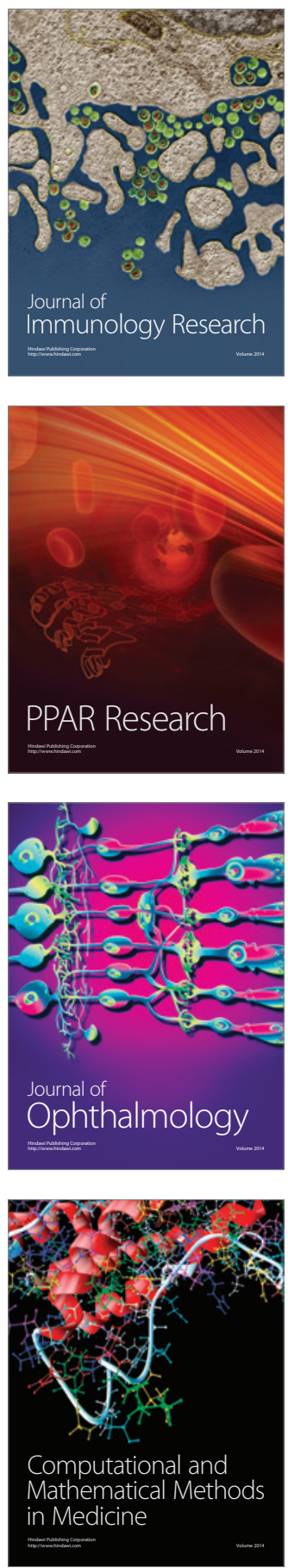

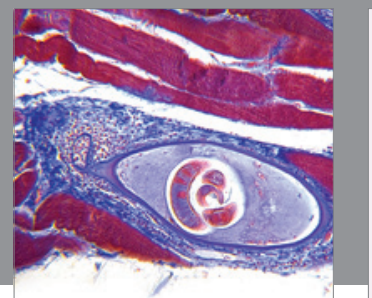

Gastroenterology

Research and Practice
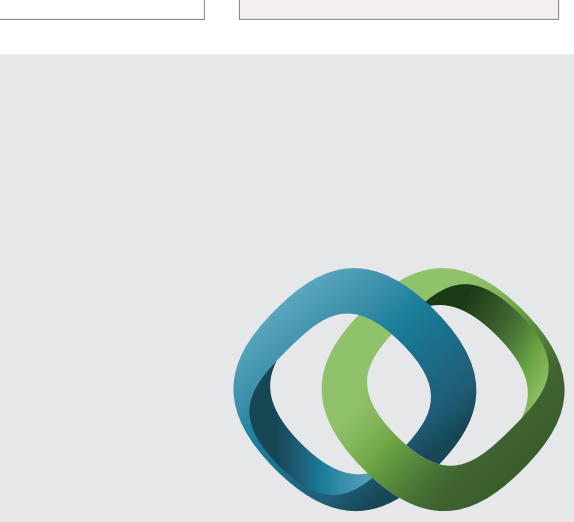

\section{Hindawi}

Submit your manuscripts at

http://www.hindawi.com
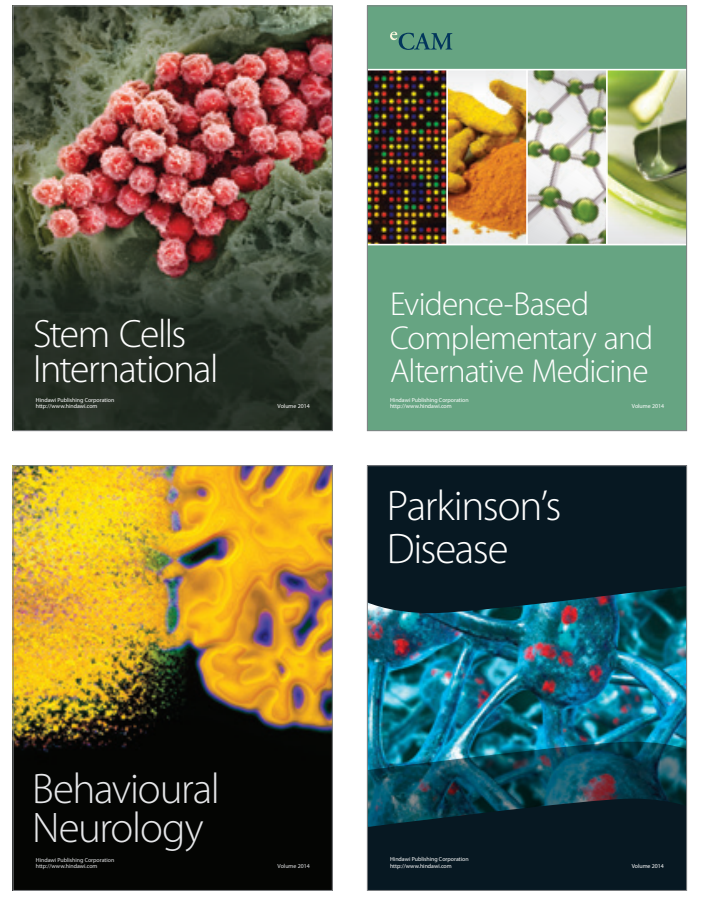
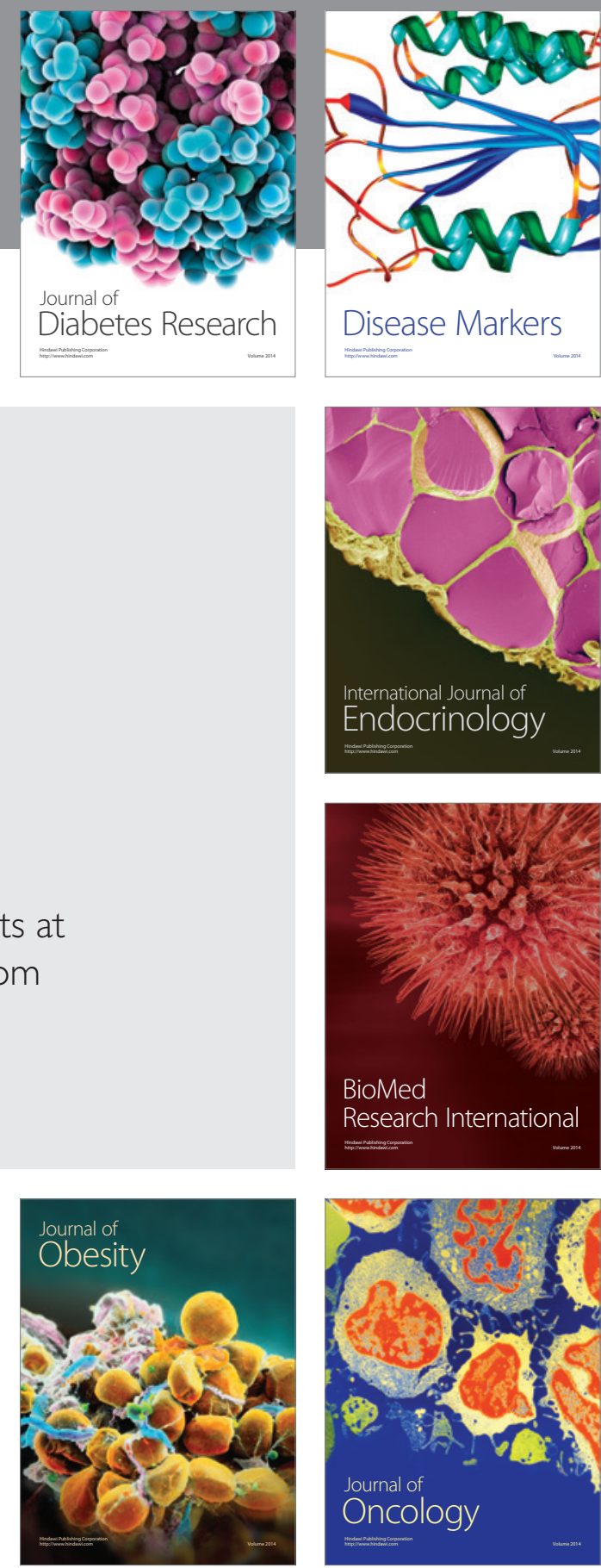

Disease Markers
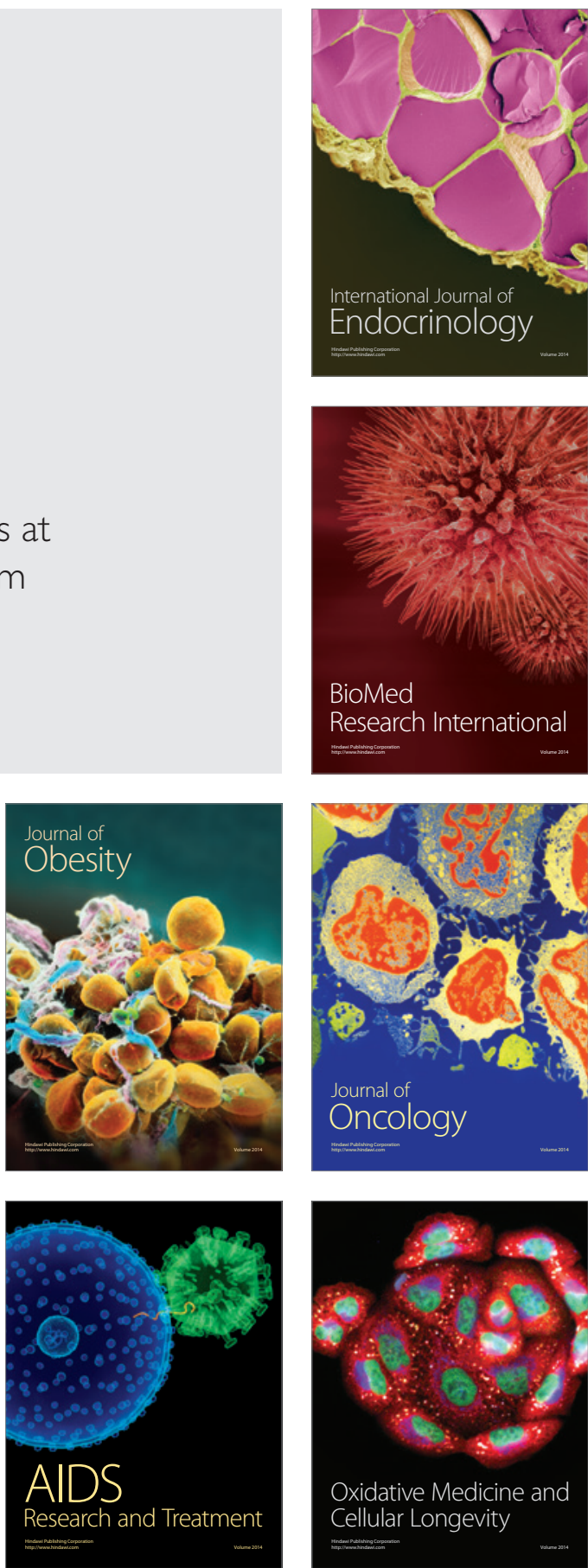